\title{
ANALYSIS OF POCKET DOUBLE GATE TUNNEL FET FOR LOW STAND BY POWER LOGIC CIRCUITS
}

\author{
Kamal K. Jha and Manisha Pattanaik \\ VLSI Design Laboratory \\ ABV- Indian Institute of Information Technology and Management, \\ Gwalior, India
}

\begin{abstract}
For low power circuits downscaling of MOSFET has a major issue of scaling of voltage which has ceased after $1 \mathrm{~V}$. This paper highlights comparative study and analysis of pocket double gate tunnel FET (DGTFET) with MOSFET for low standby power logic circuits. The leakage current of pocket DGTFET and MOSFET have been studied and the analysis results shows that the pocket DGTFET gives the lower leakage current than the MOSFET. Further a pocket DGTFET inverter circuit is design in $32 \mathrm{~nm}$ technology node at $V_{D D}=0.6 \mathrm{~V}$. The pocket DGTFET inverter shows the significant improvement on the leakage power than multi-threshold CMOS (MTCMOS) inverter. The leakage power of pocket DGFET and MTCMOS inverter are $0.116 \mathrm{pW}$ and $1.83 \mathrm{pW}$ respectively. It is found that, the pocket DGTFET can replace the MOSFET for low standby power circuits.
\end{abstract}

\section{KEYWORDS}

Pocket DGTFET, Band to Band Tunneling (BTBT), Leakage Current, MTCMOS Inverter

\section{INTRODUCTION}

With the scaling down of MOSFET, the voltage which can be applied to the gate must be reduced to maintain reliability. At the same time to maintain reliability, threshold voltage of the MOSFET has to be reduced. When gate oxide thickness reduces to about $1.2 \mathrm{~nm}$ or less, the electron tunneling occurs abruptly between gate and channel due high electric field. Power consumption goes high because of increase in sub-threshold leakage current [1-4]. To reduce leakage current alternatives were seeking, double gate MOSFET, tri gate and gate all around [5-10]. For the past few years TFET is promising candidate to overcome the issue related with MOSFET, because of less sub threshold slope (SS), lower leakage current and scaled supply voltage made this device suitable for low power applications. DGTFET shows improved characteristics including higher drive current and $57 \mathrm{mV} / \mathrm{dec}$ sub-threshold slope then single gate TFET [11-19]. Low stand by power (LSTP) devices place the accent on low voltage and low leakage devices for their operation. This application requires devices which can minimize their leakage current during sleep mode. This is also an issue with other high performance (HP) and low operating power (LOP) circuits, because sleep mode leakage power contributes to total power consumption. Conventionally the low standby power (LSTP) circuits are designed using technique of power gating in which sleep circuit is added to reduce standby leakage current by shutting off the flow of current to blocks of the circuit that are not currently in use [20]. In the present work, the design and implementation of pocket DGTFET at device as well as circuit level is carried out through the simulations and compared to the CMOS counterpart. 
International Journal of VLSI design \& Communication Systems (VLSICS) Vol.4, No.6, December 2013

\section{DeVice STRUCTURE AND Simulation}

The device structure of pocket DGTFET is shown in Fig. 1, Two-dimensional device simulations are performed for the device structure using the Version 5.11.24.C ATLAS device simulator [21]. The trivalent doping of $1 \times 10^{20} / \mathrm{cm}^{3}$ for source and pentavalent doping of $1 \times 10^{20} / \mathrm{cm}^{3}$ for drain are used. $\mathrm{HfO}_{2}$ is used as high-k dielectric (having $\varepsilon=29$ ) equivalent oxide thickness $2 \mathrm{~nm}$. Gate leakage is enabled in our simulations because of very thin gate oxide layer.

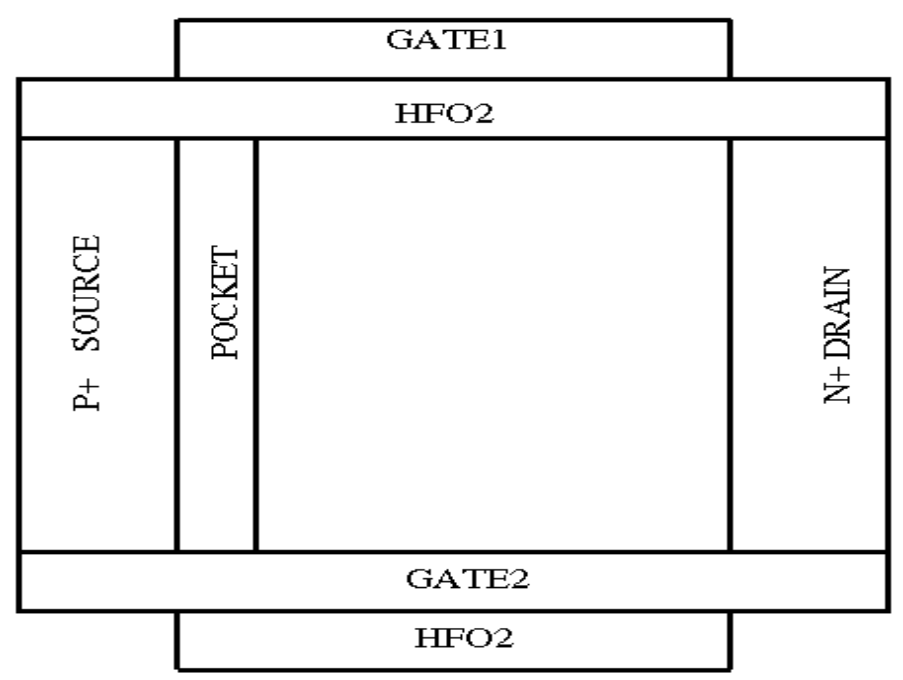

Figure 1 Device structure of pocket DGTFET.

Contacts are made of aluminum, and for gate contact, work function is set to be $4.1 \mathrm{eV}$. We have used nonlocal band to band tunneling (BTBT) model combined with a band gap narrowing model, trap assisted tunneling, shirata, mobility, quantum density-gradient and oxide tunneling model for present simulations.

\section{RESULTS AND DISCUSSIONS}

\subsection{Leakage Current of Pocket DGTFET and MOSFET}

The impact on leakage current, due to the mode of operations of both pocket MOSFET and DGTFET can be understand with energy band diagrams as shown in Fig. 2 and Fig. 3. The TFET works on the principle of band to band tunneling rather than thermionic injections of charge carriers than MOSFET counterpart. Because of BTBT in pocket DGTFET low leakage current exits as shown in Fig. 4. Applying a negative gate voltage pulls the energy bands up. A conductive channel opens as soon as the channel valence band has been lifted above the source conduction band because carriers can now tunnel into empty states of the channel. 
International Journal of VLSI design \& Communication Systems (VLSICS) Vol.4, No.6, December 2013

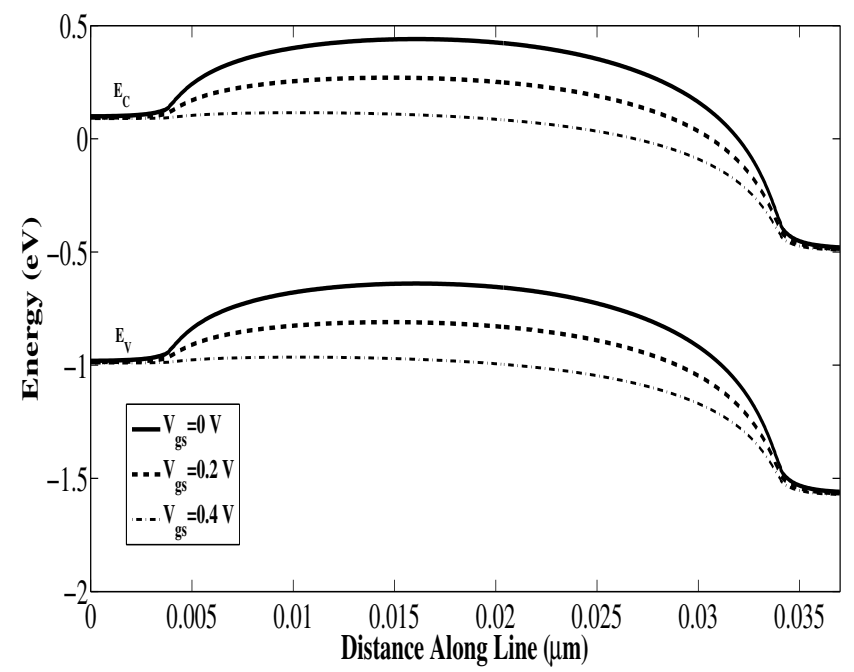

Fig. 2 Energy band diagrams of MOSFET

Because only carriers in the energy window can tunnel into the channel, the energy distribution of carriers from the source is limited; the high-energy part of the source Fermi distribution is effectively cut off. When the gate voltage exceeds the threshold voltage, the potential barrier between the channel and the source becomes narrow enough to allow a significant tunneling current, which is called ON state.

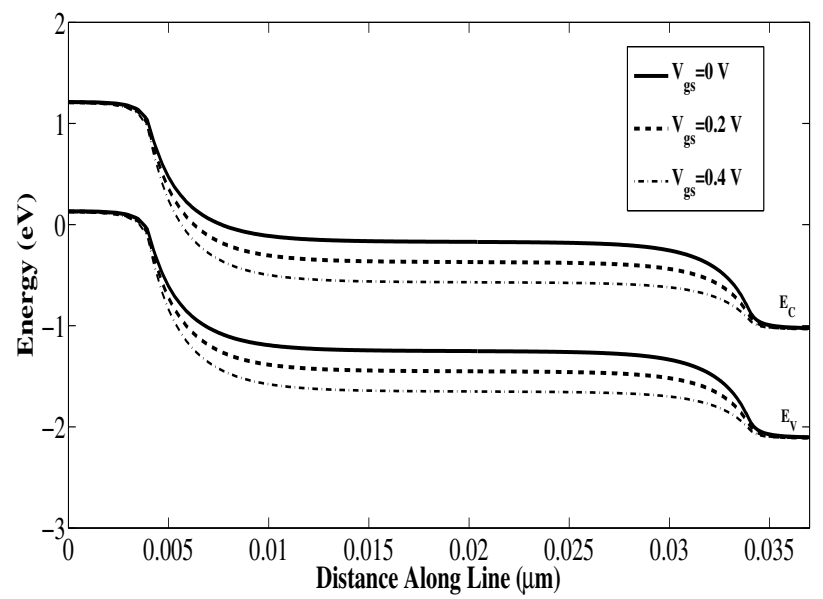

Fig.3 Energy band diagrams of pocket DGTFET

When pocket DGTFET is in its OFF state, the valence band edge of the channel is located below the conduction band edge of the source, leading to very small OFF-state currents. That are dictated by the reverse-biased $\mathrm{p}-\mathrm{i}-\mathrm{n}$ diode a given gate voltage swing compared to the MOSFETs, making the pocket DGTFET architecture an attractive to low supply voltage $\left(\mathrm{V}_{\mathrm{DD}}\right)$ digital logic circuits. 
International Journal of VLSI design \& Communication Systems (VLSICS) Vol.4, No.6, December 2013

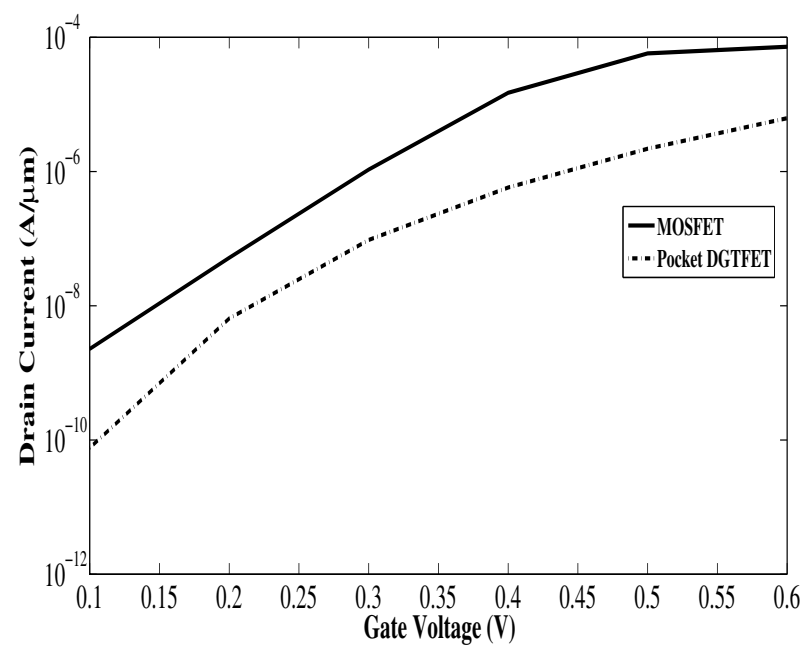

Fig. 4 Transfer characteristics (log scale) of Pocket DGTFET and MOSFET

\subsection{Design of Low leakage MTCMOS Inverter}

The leakage current is dominant factor for performance degradation of CMOS circuits. To improve the performance of CMOS circuits power gating techniques are used i.e. MTCMOS. The symbol and transient response of MTCMOS inverter are shown in Fig. 5 and Fig. 6 respectively. We have used 32nm technology node for SPICE simulations at $\mathrm{V}_{\mathrm{DD}}=0.6 \mathrm{~V}$. When sleep signal is not enabling, logic circuit will be its normal mode of operations according to input signal. As sleep signal goes high, logic block will be disconnected from power supply and data is retain their previous value because of virtual $\mathrm{V}_{\mathrm{DD}}$. In CMOS circuits sleep device is added to reduce the leakage current of circuit in OFF state. The MTCMOS inverter shows good agreement with leakage current reduction for CMOS circuits at the cost of extra circuit overhead.

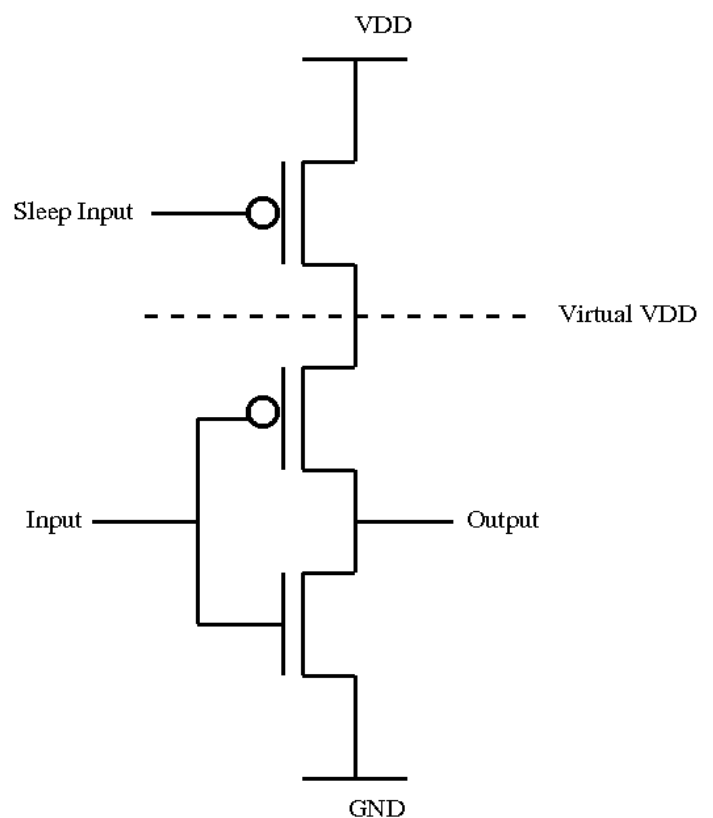

Fig. 5 MTCMOS inverter symbol 
The leakage current and leakage power of active mode of MTCMOS inverter are $1.25 \mathrm{pA}$ and $0.75 \mathrm{pW}$ respectively. During standby mode of MTCMOS inverter the leakage current and leakage power is $1.8 \mathrm{pA}$ and $3.05 \mathrm{pW}$ have been observed.

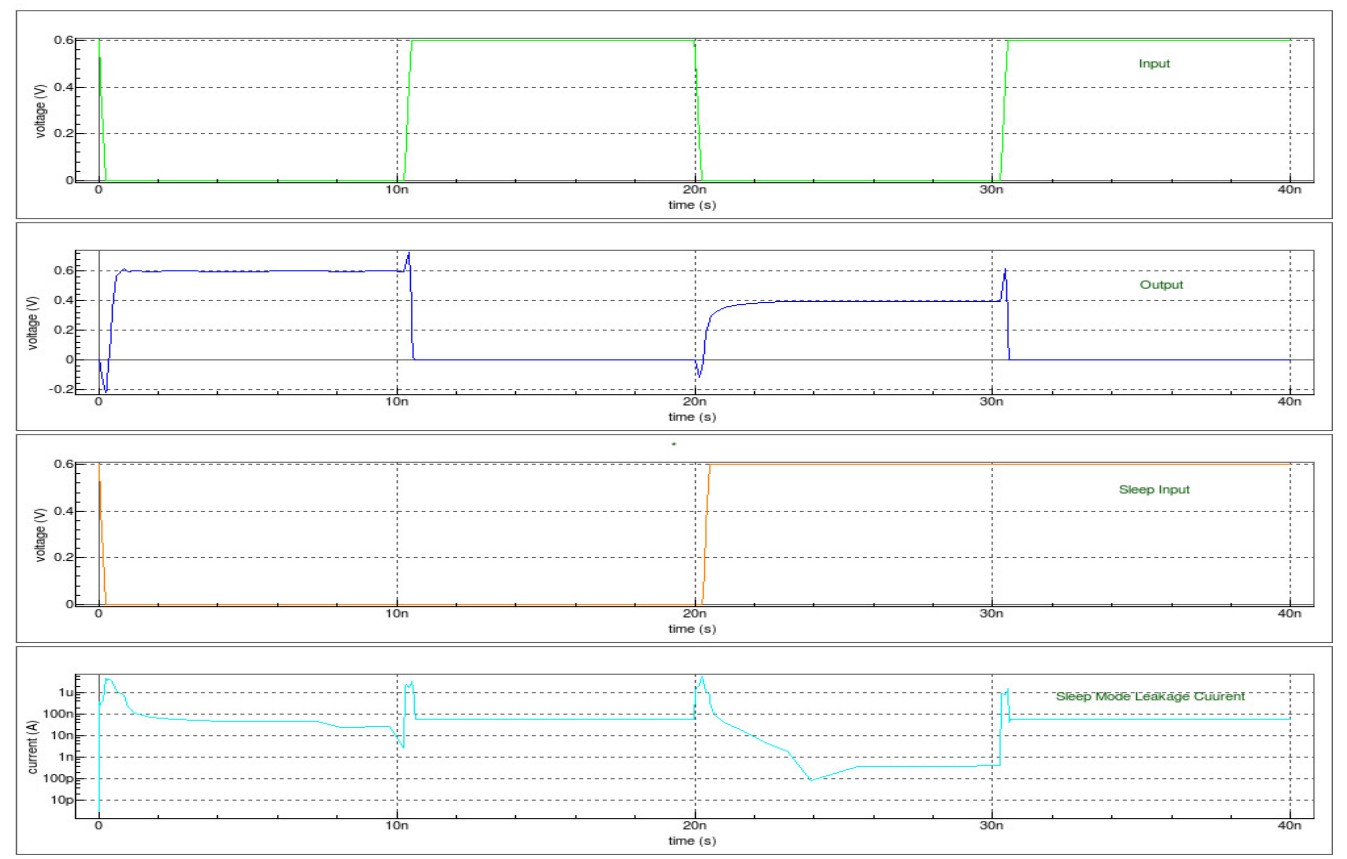

Fig. 6 Transient Response of MTCMOS inverter

It is clear from the transient response of MTCMOS inverter that, there are spikes at the output waveforms (voltage and current waveforms) during low to high or high to low transitions of input waveform. This is because of ground bounce noise i.e. when transition occurs; it takes finite time to switch from one level to other level of transition.

\subsection{Design of Low leakage Pocket DGTFET Inverter}

In order to designing a device one should have a basic idea of the parameters affecting the device and thus choose the appropriate parameters so to get the desired output and to implement the device in the required circuits. As for devices working at sub-threshold voltage region requires a sufficient amount of ON current $\left(\sim 10^{-6} \mathrm{amp}\right)$ with a minimum leakage current $\left(\sim 10^{-13} \mathrm{amp}\right)$. The ON state current of conventional TFET is not sufficient to drive the circuits, to fulfil this requirement of conventional TFET, pocket DGTFET devices are used to making the circuits. In pocket DGTFET, due to heavily doped pocket, bands are much closer at tunneling junction (i.e. reduced tunneling width). The effective tunneling area gets increased resulting higher ON current in pocket DGTFET than MOSFET counterpart. Since compact models of TFET are not available, therefore simulations are carried out using device-circuit co-design approach [21]. The symbol and transient response of Pocket DGTFET inverter are shown in Fig. 7 and Fig. 8 respectively. 
International Journal of VLSI design \& Communication Systems (VLSICS) Vol.4, No.6, December 2013

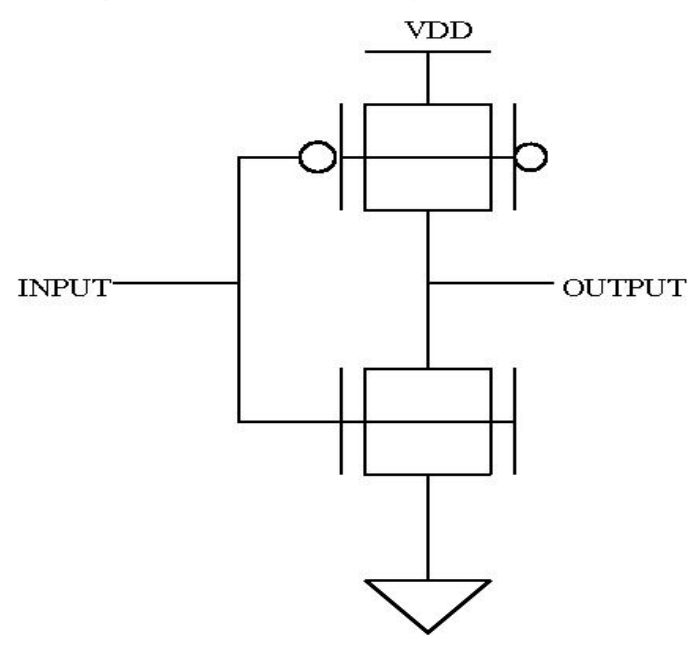

Fig. 7 Pocket DGTFET inverter symbol

The performance of pocket DGTFET inverter is compared with MTCMOS inverter and it is noticed that pocket DGTFET inverter drain less leakage current than MTCMOS counterpart.

The leakage current and leakage power of pocket DGTFET inverter are $194.4 \mathrm{fA}$ and $0.116 \mathrm{pW}$ respectively. As shown in transient analysis of pocket DGTFET, the voltage overshoot, which is expected in conventional TFET, similar behaviour is observed in pocket DGTFET. The voltage overshoot in pocket DGTFET is the consequence of larger Miller capacitance. The gate to drain capacitance $\left(\mathrm{C}_{\mathrm{gd}}\right)$ is the main dominating component of Miller capacitance in case of pocket DGTFET. However in case of MTCMOS inverter (clearly shown in Fig. 8) suffers from voltage overshoot because of ground bounce noise rather than Miller capacitance discussed above section.
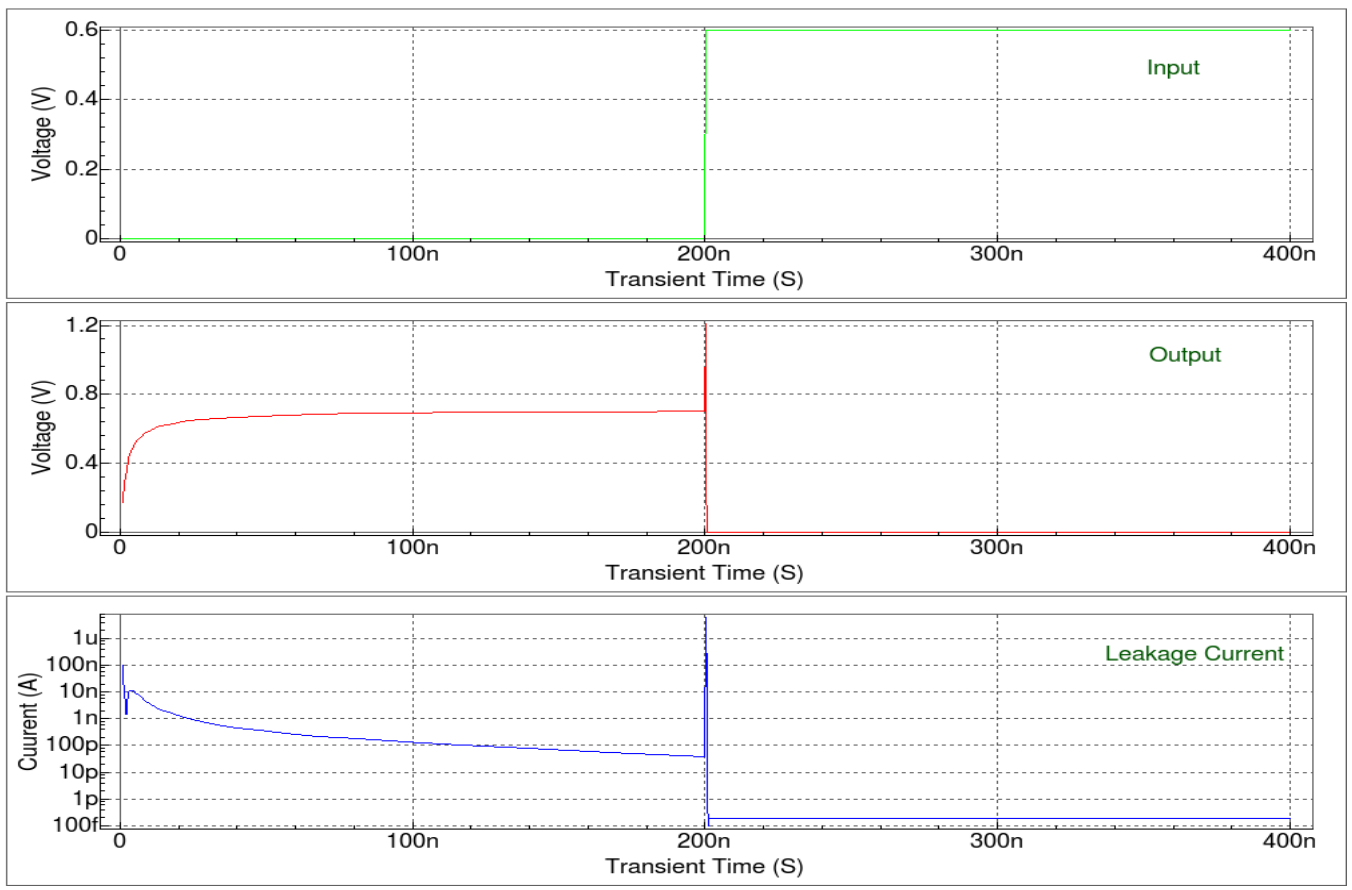

Fig. 8 Transient Response of pocket DGTFET 
International Journal of VLSI design \& Communication Systems (VLSICS) Vol.4, No.6, December 2013

\section{Conclusions}

Present work focuses the performance comparisons of pocket DGTFET for low standby power circuits. The leakage current of pocket DGTFET and MOSFET have been studied and the analysis results shows that the pocket DGTFET gives the lower leakage current than the MOSFET. Further design of Inverter circuit using pocket DGTFET and MTCMOS inverter has been discussed. The leakage current and leakage power of both pocket DGTFET and MTCMOS inverter are $194.4 \mathrm{fA}, 1.8 \mathrm{pA}$ and $0.116 \mathrm{pW}, 3.05 \mathrm{pW}$ respectively. Based on simulation results and analysis it is found that leakage power is more in MTCMOS inverter than pocket DGTFET inverter. Pocket DGTFET exhibit high performance to design low leakage logic circuits

\section{ACKNOWLEDGEMENTS}

Authors are thankful to VLSI Design Lab., ABV-Indian Institute of Information Technology and management, Gwalior, for providing the computational resources.

\section{REFERENCES}

[1] ITRS2011.[Online] http://www.itrs.net

[2] P.-J. K. Liu, "Bulk cmos scaling to the end of the roadmap", 2012

[3] A. Seabaugh, and Q. Zhang, "Low-voltage tunnel transistors for beyond cmos logic", Proceedings of the IEEE, Vol. 98, No.12, 2010

[4] A. M. Ionescu, and H. Riel, "Tunnel field-effect transistors as energy-efficient electronic switches", Nature, Vol. 479, 2011

[5] K. Boucartand, and A. M. Ionescu, "Double-gate tfet with high- $\kappa$ gate dielectric", IEEE Trans. on Elec. Dev., Vol. 54, No. 7, 2007

[6] C. Anghel, P. Chilagani, A. Amara, and A. Vladimirescu, "Tunnel field effect transistor with increased ON current, low-k spacer and high-k dielectric", Appl. Phys Lett. Vol. 96, No. 12, 2010

[7] C. Anghel, A. Gupta, A. Amara, and A. Vladimirescu, "30-nm TFET with improved performance and reduced ambipolar current", IEEE Tran. on Elec. Dev., Vol. 58, No.6, 2011

[8] S. Agarwal, and E. Yablonovitch, "Enhanced tunneling current in id-edge overlapped tfet's", 70th Annual Device Research Conference(DRC), 2012

[9] U. E. Avci, R. Rios, K. Kuhn, and I. A. Young, "Comparison of performance, switching energy and process variations for the tfet and MOSFET in logic”, Symposium on VLSI Technology Digest, 2011

[10] R. Jhaveri, V. Nagavarapu, and J. C. S. Woo, "Effect of pocket doping and annealing schemes on the source-pocket tunnel field-effect transistor", IEEE Trans. on Elec. Dev., Vol. 58, No. 1, 2011

[11] A. Mallik, and A. Chattopadhyay, "The impact of fringing field on the device performance of a pchannel tunnel field-effect transistor with a high-k gate dielectric", IEEE Trans. on Elec. Dev., Vol. 59, No. 2, 2012

[12] L. D. Michielis, L. Lattanzio, P. Palestri, L. Selmi, and A. Ionescu, "Tunnel-fet architecture with improved performance due to enhanced gate modulation of the tunneling barrier", 69th Annual Device Research Conference (DRC), 2011

[13] W. Y. Choi, B.-G Park, J. D. Lee, and T.-J. K. Liu, "Tunneling field-effect transistors (tfets) with subthreshold swing (ss) less than 60 mv/dec", IEEE Elec. Dev. Lett., Vol. 28, No.8, 2011

[14] A. Chattopadhyay, and A. Mallik, "Impact of a spacer dielectric and a gate overlap/underlap on the device performance of a tunnel field-effect transistor", IEEE Trans. on Elec. Dev., Vol. 58, No. 3, 2011

[15] S. Koswatta, M. Lundstrom, and D. Nikonov, "Performance comparison between p-i-n tunneling transistors and conventional mosfets", Electron Devices, IEEE Trans. on Elec. Dev., Vol. 56, No. 3, 2009

[16] W. Lee, and W. Choi, "Influence of inversion layer on tunneling field-effect transistors", IEEE Elec. Dev. Lett., Vol. 32, No. 9, 2011 
International Journal of VLSI design \& Communication Systems (VLSICS) Vol.4, No.6, December 2013

[17] Y. Lu, A. Seabaugh, P. Fay, S. J. Koester, S. Laux, W. Haensch, and S. O. Koswatta, "Geometry dependent tunnel fet performance - dilemma of electrostatics vs. quantum confinement", Device Research Conference (DRC), 2010

[18] A. Mallik, and A. Chattopadhyay, "Drain-dependence of tunnel field-effect transistor characteristics: The role of the channel", IEEE Trans. on Elec. Dev., Vol. 58, No. 12, 2011

[19] A. Pal, B. Sachid, H. Gossner, and V. R. Rao, "Insights into the design and optimization of tunnel-fet devices and circuits", IEEE Trans. on Elec. Dev., Vol. 58, No. 4, 2011

[20] R. G. Dreslinski, M. Wieckowski, and D. Blaauw, "Near-threshold computing: reclaiming moore's law through energy efficient integrated circuits", Proceedings of the IEEE Vol. 98, No. 2, 2010

[21] Atlas User's Manual, Silvaco Int., Santa Clara, CA, 2013 